Sinergias educativas

ISSN: 2661-6661

compasacademico@icloud.com

Grupo Compás

Ecuador

\title{
Enseñanza y evaluación de la competencia ortográfica en primaria
}

Palacios Heredia, Jéssica Mariela

Enseñanza y evaluación de la competencia ortográfica en primaria

Sinergias educativas, vol. 2, núm. 2, 2017

Grupo Compás, Ecuador

Disponible en: http://www.redalyc.org/articulo.oa?id=573561679006

Esta obra está bajo una Licencia Creative Commons Atribución-NoComercial-SinDerivar 4.0 Internacional. 


\title{
Enseñanza y evaluación de la competencia ortográfica en primaria
}

\author{
Teaching and evaluation of spelling proficiency in primary \\ Jéssica Mariela Palacios Heredia \\ angelicamendoza@gmail.com \\ Profesora Unidad Educativa Nueva Semilla, Ecuador \\ (D) http://orcid.org/0000-0003-0527-2649
}

Sinergias educativas, vol. 2, núm. 2, 2017

Grupo Compás, Ecuador

Recepción: 15 Marzo 2017

Aprobación: 25 Noviembre 2017

Redalyc: http://www.redalyc.org/ articulo.oa?id $=573561679006$

\section{BY-NC-ND}

Resumen: Tenemos como objetivo el ana\#lisis de una realidad en nuestras aulas de clase: las faltas ortogra\#ficas en los escritos de los estudiantes. Mediante entrevistas planteadas a los docentes de nuestra institucio\#n, "Nueva Semilla" hemos concluido que son varios los factores que influyen en el nivel acade\#mico ortogra\#fico, empezando por la necesidad de que sean docentes y padres de familia los modelos de escritura y de estudio a seguir por nuestros pequen\#os, mediante el ejercicio y la pra\#ctica de una cultura lectora. Por nuestra investigacio\#n afirmamos que es necesario implementar en la planificacio\#n actual estrategias para ensen\#ar, -como la memoria visual- e instrumentos para evaluar, potenciar en los docentes su creatividad, incluyendo uso las TICs en las clases para desarrollar en los estudiantes el deseo de cultivar y mantener la conciencia ortogra\#fica. El estudio es relevante porque ahora estamos al tanto de lo que hemos logrado y lo que falta por hacer adema\#s es un referente.

Palabras clave: Ortografi\#a, me\#todo de aprendizaje, criterios de evaluacio\#n, dida\#ctica.

Abstract: We aim to analyze a reality in our classrooms: spelling mistakes in students' writings. Through interviews with teachers of our institution, "Nueva Semilla" we have concluded that there are several factors that influence the orthographic academic level, starting with the need for teachers and parents to be the models of writing and study to follow for our little ones, through the exercise and practice of a reading culture. Through our research we affirm that it is necessary to implement in the current planning strategies to teach, -like visual memory- and instruments to evaluate, strengthen teachers in their creativity, including using ICTs in classes to develop in students the desire to cultivate and maintain spelling awareness. The study is relevant because we are now aware of what we have achieved and what remains to be done is also a reference.

Keywords: Spelling, learning method, evaluation criteria, teaching.

\section{INTRODUCCIÓN}

Co\#mo en todas las instituciones de Educacio\#n Ba\#sica, los maestros de "Nueva Semilla" tambie\#n estamos ante el desafi\#o de despertar y mantener la conciencia ortogra\#fica de los estudiantes desde segundo a se\#ptimo an\#o de la Educacio\#n General Ba\#sica. Sen\#alamos como posibles causas del problema el desintere\#s y la poca pra\#ctica en escritura y redaccio\#n; la falta de estimulacio\#n para despertar la inclinacio\#n hacia la lectura; la pobreza lexical de los estudiantes, su dificultad para captar el sentido de lo que leen y escuchan y el escaso desarrollo de la memoria visual, y la necesidad de que el personal docente sea ma\#s recursivo a la hora de desarrollar el "aparato ortogra\#fico" en clases. 
Creemos que si se desarrolla la memoria visual consciente, mejorara\# el ejercicio ortogra\#fico escolar.

El trabajo es original porque nosotros no contamos con un estudio que indique co\#mo lograr que las nuevas generaciones, llamadas digitales, alcancen un nivel deseable en el a\# rea de la ortografi\#a. Se incluyen los resultados de entrevistas a maestros con experiencia docente en la ensen\#anza de Lengua y Literatura de nuestra escuela que sen\#alan aportaciones pra\#cticas para la ensen\#anza y valoracio\#n de la ortografi\#a en el aula.

El estudio se realizo\# con el referente bibliogra\#fico hallado en revistas revisadas en los buscadores Scielo y Redalyc.

La Serie Galileo para el Aprendizaje de la Ortografi\#a de la Revista de Investigacio\#n Educativa 19 de CPU-e sen\#ala que la ortografi\#a no es cuestio\#n de reglas ni de normas, sino de costumbres y es eso lo que tienen que entender los estudiantes cuando se les ensen\#a a escribir (no la grama\#tica como se confunde usualmente) porque su finalidad no es otra sino la de facilitar la comunicacio\#n.

Este estudio, es importante porque analiza el desarrollo de las habilidades comunicativas y de competencias lectoras, escritas y orales en los escolares, con miras a la formacio\#n de una cultura amiga de la consulta de libros, que enriquezca su vocabulario y mejore el ejercicio ortogra\#fico.

Establece un referente del momento en que debe empezar el aprendizaje de lecto-escritura y co\#mo fortalecer las

habilidades lectoras y escritas, en los estudiantes, contribuir en el ana\#lisis para el mejoramiento del aprendizaje de la ortografi\#a en el aula. Resalta la importancia de su incorporacio\#n co\#mo asignatura de aula. Con esta investigacio\#n daremos respuesta a las siguientes preguntas: ¿A que\# edad se debe ensen\#ar ortografi\#a? ¿Que\# metodologi\#a aplicada resulta la ma\#s ido\#nea para su ensen\#anza?

¿Podemos construir en cada cuaderno del estudiante un apartado para trabajar exclusivamente la ortografina?, co\#mo se haci\#a en la escuela tradicional. $\mathrm{Y}$ ¿co\#mo nos ha ido al respecto en nuestra institucio\#n? ¿Que\# me\#todos usan en su pra\#ctica docente nuestros maestros? ¿Cua\#les son los instrumentos de evaluacio\#n y estrategias para la ensen\#anza de la ortografi\#a en la UENS?

Empezaremos definiendo que\# es la Ortografi\#a.

La Real Academia de la Lengua Espan\#ola en su texto de Ortografi\#a (2010), manifiesta que: La ortografi\#a de una lengua no es una mera amalgama de reglas, sino que constituye un sistema estructurado, que se articula en varios subsistemas dotados de cierta autonomi\#a, constituidos por las normas que regulan de forma especi\#fica el uso de cada una de las clases de signos gra\#ficos con que cuenta su sistema de escritura. Todos los sistemas ortogra\#ficos poseen un subsistema de

10 reglas que determina el valor de las letras o grafemas en la representacio\#n gra\#fica de las unidades le\#xicas, al ser los grafemas los constituyentes primarios, y durante mucho tiempo u\#nicos, de todos los sistemas de escritura. (pa\#g. 11). 
Importancia del estudio de la Ortografi\#a co\#mo asignatura en la escuela ecuatoriana

Comparemos opiniones de dos expertos sobre la materia: Segu\#n Vaca Uribe aprender la ortografi\#a de una lengua es aprender co\#mo escriben los miembros de una comunidad lingu\#i\#stica. Para este autor, la escritura es un medio de comunicacio\#n que esta\# fuertemente ligado a la lengua hablada, pero que no depende enteramente de ella. No escribimos como hablamos. El espan\#ol es la lengua que hablamos y escribimos en Me\#xico, Espan\#a y Latinoame\#rica, entre otros muchos pai\#ses y regiones del mundo. Cerca de 400 millones de seres humanos hablamos espan\#ol. Aunque todos hablemos espan\#ol, entre los diferentes pai\#ses y entre las diferentes regiones de cada pai\#s, las personas hablamos ligeramente diferente unas de otras: usamos algunas palabras diferentes para decir lo mismo o usamos las mismas palabras, pero las pronunciamos un poco diferente; usamos diferente entonacio\#n o a veces usamos en un orden diferente las mismas palabras, etc. Por esas diferencias, la escritura no puede ser simplemente el dibujo de lo que hablamos.

En contraposicio\#n, a Ja\#uregui Olaza\#bal

le tiene sin cuidado la buena ortografi\#a, lo que le preocupa es que sus lectores le comprendan y lo que deben ensen\#ar los maestros a sus alumnos es que se entiendan hablando.

Ambos autores han estudiado la influencia que la ensen\#anza de la ortografi\#a co\#mo asignatura tiene en los estudiantes, sin embargo, Vaca y Jau\#regui concuerdan en que la forma co\#mo escribimos tiene un impacto en nuestra forma de comunicarnos y en la imagen que proyectamos a los dema\#s.

¿Y los ecuatorianos que\# pensamos sobre la importancia de escribir correctamente?

No han sido pocas las veces que al escribir una palabra hemos dudamos sivaconsoc,sivaconvob,sise tilda o no. ¿En cua\#ntas ocasiones nos ha tocado preguntar o buscar un diccionario para asegurarnos de que escribimos bien? En nuestro pai\#s las propiedades de nuestra lengua tienen un valor significativo.

No es raro leer en los diarios de mayor circulacio\#n nacional, apartados dedicados al uso del idioma, las u\#ltimas aceptaciones de la RAE, expresiones idioma\#ticas y au\#n nuestra memoria evoca el recuerdo del segmento que apareci\#a en televisio\#n nacional "Sabe usted la palabra correcta" con Juanita Vallejo, por tanto; podemos concluir que el uso de los elementos de la lengua (grama\#tica, morfologi\#a, sema\#ntica, caligrafi\#a, ortografi\#a, entre otros) siempre han sido significativos y tradicionalmente, -podemos asegurarlo, por lo que explica la Actualizacio\#n y Fortalecimiento Curricular 2010- han sido los aspectos a los que ma\#s se les

ha dado importancia en la planificacio\#n curricular ecuatoriana.

Debido a ese intere\#s, el enfoque comunicativo de la escuela ecuatoriana actual plantea que la ensen\#anza de la lengua debe centrarse en el desarrollo de las habilidades y conocimientos necesarios para comprender y producir eficazmente mensajes lingu\#i\#sticos en distintas 
situaciones de comunicacio\#n, y para lograrlo adema\#s de leer bien, debemos escribir bien y por eso la ortografi\#a forma parte del A\#rea de Lengua en la Educacio\#n Ba\#sica.

Este juicio de valor es el que deben tener nuestros estudiantes de primaria, especialmente porque son los que han desarrollado su propia ortografi\#a y forma de comunicarse en las redes sociales con sus dispositivos electro\#nicos, dejando de lado por instantes, la correcta escritura de las palabras, que con cada di\#a que pasa, se vuelve menos importante en las generaciones jo\#venes.

La revista Algunas confusiones en Lingu\#i\#stica aplicadas y sus consecuencias explica que los estudiosos sugieren a los docentes ensen\#ar las diferencias entre la comunicacio\#n oral y escrita para desarrollar la conciencia ortogra\#fica: "Es necesario concienciar a los maestros y a quienes tienen responsabilidad directa (...) de las diferencias sustanciales que separan la comunicacio\#n escrita de la comunicacio\#n oral”. Sin embargo, por una desafortunada tradicio\#n, la atencio\#n y el desarrollo del co\#digo oral han sido abandonados por la escuela. Efectivamente, la oralidad

ha sido ocupacio\#n del hogar, en tanto que la escritura ha sido quehacer de la escuela. En los te\#rminos de Prado la situacio\#n ha sido la siguiente:

Las habilidades orales tradicionalmente han estado desatendidas y no han sido objeto de ensen\#anza, por entender que las mismas las desarrollaba el nin\#o a partir de su aprendizaje en el entorno familiar y social y, por tanto, no era necesaria su ensen\#anza, pues el nin\#o cuando llegaba a la escuela ya sabi\#a hablar y escuchar (2004: 145).

A la vista de este consenso no deberi\#a existir problema alguno en asegurar que uno de los objetivos esenciales de la clase de lengua consiste en lograr que el estudiante produzca los textos que la sociedad le demande. De igual modo, cabri\#a tambie\#n pensar que la escuela ha logrado que los estudiantes dominen la escritura. Sin embargo, cabe preguntamos: ¿Cua\#ndo empezar?

Desarrollo de la conciencia ortogra\#fica Muchos padres opinan, erro\#neamente, que la educacio\#n de los hijos empieza cuando e\#stos ingresan en el jardi\#\# de la infancia o en el primer an\#o de Educacio\#n Ba\#sica. El hecho es que el cerebro del nin\#o, parecido a un ordenador, comienza a funcionar desde el momento del nacimiento, y simplemente esta\# esperando que se le alimente con informacio\#n que pueda

almacenar y usar en el futuro. De modo que podemos empezar a "programarlo" desde el nacimiento. Descartemos la idea erro\#nea de que solo un diplomado puede ayudarnos a lograrlo. Investigaciones extensas han

mostrado que el grado de e\#xito que el nin\#o alcanza en logros como los de aprender a hablar y leer esta\#, "segu\#n se cree, estrechamente relacionado con la clase de interaccio\#n que exista entre los padres y el nin\#o durante la tierna infancia de e\#ste, el grado de intere\#s que los padres le muestren, y el que ellos puedan animarlo a explorar por si\# mismo en su ambiente". 
Por tanto tenemos "tesoros valiosos" en nuestras manos que queremos aprovechar mediante el esti\#mulo temprano y el desarrollo de la competencia de lecto-escritura en la que desde el mismo inicio esta\# inmersa la ortografi\#a.

Del ana\#lisis de la revista "Las habilidades fonolo\#gicas, su organizacio\#n neurofisiolo\#gica y su aplicacio\#n en la educacio\#n", escribimos que el aprendizaje de la lectura debe considerar aspectos pedago\#gicos, psicolingu\#i\#sticos, neuropsicolo\#gicos y fisiolo\#gicos (Feld, y Rodri\# guez, 2004). Pues esta tarea se enmarca en un contexto social determinado, en un proceso de creciente comunicacio\#n por parte de los nin\#os entre si\# y con los adultos. El nin\#o ingresa al preescolar y al primer grado con un bagaje de conocimientos que le permite expresarse de determinada forma y con un le\#xico relativamente desplegado, entiende ciertas inflexiones proso\#dicas, y usa determinada sintaxis. En esta primera etapa predominan los aspectos neurofisiolo\#gicos correspondientes a la lectura facilitada, que se caracteriza por la accio\#n de diversos esti\#mulos como

los emergentes de los estereotipos fonema\#ticos (aspectos fonolo\#gicos del lenguaje).

Este conocimiento lingu\#i\#stico del nin\#o facilita el reconocimiento del grafema, el cual se apoya en el descubrimiento esponta\#neo de relaciones entre el lenguaje oral y escrito (relacio\#n entre fonema y grafema), el uso de semejanzas visuales (palabras con formas similares), la adivinacio\#n y la orientacio\#n del adulto. A su vez, el aprendizaje de la escritura permite el reforzamiento de la relacio\#n entre grafema y fonema, que puede escribir y pronunciar simulta\#neamente. No se considera menor el papel del aspecto sema\#ntico de la lectura producido por un cierto grado de facilitacio\#n que ello produce y que caracteriza el conocimiento que el nin\#o posee desde su infancia. Como sen\#ala Azcoaga et al. (1997), se trata de un proceso oscilante y progresivo.

Por lo investigado, concluimos que para desarrollar la macrodestreza: escribir, el profesorado debera\# trabajar con las microhabilidades que se involucran en los procesos de la lengua, de manera progresiva, sistema\#tica y recursiva durante toda la Educacio\#n General Ba\#sica comenzando con la alfabetizacio\#n, pero tambie\#n desde la necesidad de comunicar.

Otros expertos puntualizan en que como asignatura la ensen\#anza de la ortografi\#a se debe iniciar cuando el dominio de la escritura sea claro y fluido, ma\#s o menos a los ocho an\#os de edad. Concuerdan en que se debe priorizar las palabras de mayor uso y mayor problema ortogra\#fico y que a partir de

los doce an\#os se puede comenzar un trabajo de profundizacio\#n, ya que este es el momento adecuado para estudiar las reglas ortogra\#ficas.

Y ¿co\#mo nos ha ido al respecto en nuestra institucio\#n? ¿Que\# me\#todos usan en su pra\#ctica docente nuestros maestros? ¿Cua\#les son las particularidades de evaluacio\#n y de ensen\#anza de la ortografi\#a en la UENS?

Objetivos de la investigacio\#n La investigacio\#n aqui\# resen\#ada tuvo 
como objetivo principal analizar las aportaciones de los docentes en la ensen\#anza, correccio\#n y calificacio\#n de la ortografi\#a, considerando la importancia que ellos atribuyen a los siguientes aspectos: Grado de dificultad que presentan los estudiantes de la seccio\#n Primaria de la Unidad Educativa "Nueva Semilla", metodologi\#a para la ensen\#anza ortogra\#fica que actualmente se esta\# utilizando en nuestra escuela, me\#todos nuevos que llevara\#n a superar las debilidades que a nivel ortogra\#fico se presentan.

Intere\#s que muestran los alumnos, en la ortografi\#a de las letras, tildes y el uso correcto de los signos de puntuacio\#n al momento de escribir o redactar y la creacio\#n de un Manual de Ortografi\#a que mejorara\# la comunicacio\#n escrita en los estudiantes de la Unidad "Nueva Semilla".

\section{MATERIALES Y MÉTODOS}

El trabajo de investigacio\#n se realizo\# en

la Unidad Educativa "Nueva Semilla”, con los maestros de primaria, la muestra seleccionada fue conformada

por las seis maestras de Lengua y Literatura de $2^{\circ}$ a $7^{\circ}$ an\# os EGB.

Se utilizo\# una metodologi\#a cualitativa. Se disen\#o\# en base a la revisio\#n que se efectu\#a en cuadernos y libros trabajados por los estudiantes en las visitas a\#ulicas mediante entrevistas dirigidas a docentes con i\#tems impresos como preguntas abiertas relacionadas a las cuestiones anteriormente citadas. Este cuestionario fue llenado por el grupo de maestras encargadas de la elaboracio\#n del plan semanal de destrezas de cada an\#o de Ba\#sica, ya que en nuestra institucio\#n se lo hace por a\#reas; se adiciono\# un apartado en el que se pregunto\# a los docentes que\# recomiendan a sus estudiantes para evitar faltas ortogra\#ficas en sus escritos y que\# criterios utilizan para evaluar los textos de estos u\#ltimos.

Se tuvo mucho cuidado en no influenciar de modo alguno en las respuestas de los docentes, el encuentro fue flexible y holgado ya que se buscaron espacios en las horas libres de las compan\#eras quienes se sintieron libres de incluir en la entrevista algunas experiencias a\#ulicas que reflejaron el ejercicio diario del desarrollo de la competencia ortogra\#fica. Esta investigacio\#n puede ser un referente para pro\#ximos trabajos relacionados con la destreza ortogra\#fica.

\section{RESULTADOS}

En las entrevistas fueron identificados 7 segmentos correspondientes al trabajo a\#ulico de los docentes en nuestra institucio\#n. A partir de estos, resultan cuatro categori\#as tema\#ticas que reflejan la apreciacio\#n de los maestros respecto a las dificultades en el a\#rea y a la metodologi\# a aplicada

familia Uso del diccionario

Sugerencias para motivar el aprendizaje dina\#mico de la ortografi\#a Aplicar te\#cnicas cacogra\#ficas Monitoreo constante 
Puntos extras y stickers Uso de las TICS Adicionalmente las maestras recomendaron construir en cada cuaderno del estudiante un apartado para trabajar exclusivamente la ortografi\#a.

\section{DISCUSIÓN}

El objetivo de esta investigacio\#n fue analizar las aportaciones de los docentes en su ejercicio diario de ensen\#anza y de evaluacio\#n de la ortografi\#a frente a la problema\#tica presentada en la escuela primaria. $\mathrm{Al}$ respecto, los resultados ponen en evidencia ciertas particularidades en las pra\#cticas pedago\#gicas descritas; tambie\#n se logro\# determinar en te\#rminos generales que la ortografi\#a en el estudiantado no es un tema de mucho intere\#s, especialmente entre los ma\#s pequen\#os. Lo que esta\# en armoni\#a con lo que explica la Programacio\#n Neurolingu\#i\#stica: la conciencia ortogra\#fica se establece a los doce an\#os de edad, comprobo\# que la falta de estimulacio\#n para despertar la inclinacio\#n hacia la lectura, la pobreza lexical de los estudiantes, el escaso desarrollo de la memoria visual, y la necesidad de que el personal docente sea ma\#s recursivo a la hora de desarrollar el "aparato ortogra\#fico" en clases si son causales determinantes para el desarrollo de la competencia ortogra\#fica.

Dado que la evaluacio\#n del aprendizaje constituye el proceso de mayor complejidad en el quehacer educativo, se requiere valorar el desarrollo y cumplimientos de los objetivos de la ensen\#anza de ortografi\#a. Notamos que nuestros docentes deben incrementar el nivel de conocimiento sobre instrumentos de evaluacio\#n ortogra\#fica incluyendo una evaluacio\# $\mathrm{n}$ diagno\#stica continua que detecte a tiempo las insuficiencias y limitaciones de los estudiantes, con el objetivo de implementar las medidas correctivas que requieran tanto la ensen\#anzaaprendizaje como la evaluacio\#n.

\section{CONCLUSIONES}

Como docentes a diario palpamos lo que la realidad revela: muchi\#simos estudiantes de primaria no escriben ni leen al nivel que les es esencial para que efectu\# en con e\#xito sus tareas acade\#micas. Es lo\#gico, entonces, que el dominio de una aptitud tan esencial en la vida deba procurarse con la mayor prontitud. Por lo tanto, para padres y docentes deberi\#a ser de importancia primordial ensen\#ar a sus nin\#os a hacerlo. Ya que la ortografi\#a no solo se ensen\#a, sino que tambie\#n se aprende.

El aporte que damos con nuestra investigacio\#n apunta a que los docentes entrevistados configuran sus pra\#cticas de ensen\#anza y de evaluacio\#n de la ortografi\#a, creyendo importante

el uso de las TICs y de estrategias que desarrollen la memoria visual, considerado como el mejor me\#todo para ensen\#ar ortografi\#a a partir de los ocho an\#os, sin olvidar que la estimulacio\#n temprana es importante desde la primera infancia. Los resultados indican que conocemos pocos me\#todos para ensen\#ar ortografi\#a, por tanto, debemos seguir 
investigando otras estrategias que se implementen en los salones para "llegar" a los estudiantes y motivarlos ma\#s que todo, a acrecentar en ellos el "gusto" por escribir bien. Tambie\#n queremos considerar la posibilidad de que los docentes del a\#trea generen un manual pra\#ctico y de uso sencillo, luego de investigar estrategias dina\#micas y actuales. Esta investigacio\#n no respondio\# que\# es ni en que\# consiste el me\# todo VOCEP, tampoco demostro\# co\#mo contrarrestar la incidencia que el "le\#xico" virtual juvenil actual tiene en la expresio\#n oral y escrita de nuestros nin\#os.

\section{Referencias}

Vaca Uribe, J. (2014). Serie Galileo para el Aprendizaje de la Ortografi\#a. Revista CPU-e. 19(1), 278-318.

Feld, V. (2014). Las habilidades fonolo\#gicas, su organizacio\#n neurofisiolo\#gica y su aplicacio\#n en la educacio\#n. Pensamiento Psicolo\#gico. 12 (1) 71-82.

Escorcia, D., Moreno, M., Campo, K., \& Palacio, J. (2014). Ensen\#anza y evaluacio\#n de la escritura en la universidad: ana\#lisis de pra\#cticas declaradas de docentes franceses y colombianos. Revista del Instituto de Estudios en Educación del Norte. 20(1), 92-107.

Ja\#imez,R., \& Maurera, S.(2009). Algunas Confusiones En Lingu\#i\#stica Aplicada Y Sus Consecuencias. Revista de lingu\#i\#stica teo\#rica y aplicada. $47(2), 35-50$

Riestra, D. (2007). Los textos como acciones de lenguaje, un giro epistemolo\# gico en la dida\#ctica de la lengua. Co-herencia. 4(7)1-15 